Den gesetzlichen Anforderungen nicht entsprechende Proben:

Mit einer Verwarnung zugelassen in Fällen erstmaligen Verstoßes............ . . Zugelassen, nach Abänderung der Aufschriften in Gemäßheit des Gesetzes . . . . . . . . . . Zurückgewiesen mit der Aufforderung, die Waren aus dem Gebiete der Vereinigten Staaten wieder auszuführen .. . . . . . . . . .

Abgewiesen, jedoch nicht vernichtet ...... Insgesamt : Den gesetzlichen Anforderungen entsprechende Proben. . . . . . . . . . . . . . . . .

Gesamtzahl der untersuchten Proben . . . . . .
Wein

Fleisch Olivenöl Vers

erschie-

zu.

sammen

\begin{tabular}{rrrrr}
50 & 9 & 11 & 38 & 108 \\
1 & 9 & 10 & 17 & 37 \\
37 & 2 & 14 & 3 & 56 \\
4 & 11 & 2 & 5 & 32 \\
\hline 92 & 31 & 37 & 63 & 223 \\
776 & 150 & 476 & 255 & 1657 \\
\hline 868 & 181 & 513 & 318 & 1880
\end{tabular}

geteilt worden sind, indessen ist dadurch die gehörige Durchführung des Gesetzes in keiner Weise beeinträchtigt worden. Auch ist es erfreulich, daß die Exporteure im Auslande in der Regel bestrebt gewesen sind, den genauen Charakter der gesetzlichen Vorschriften kennen zu lernen, und in vielen Fällen sich ernstlich bemüht haben, denselben nachzukommen. Nichtsdestoweniger liegen, wie überall, so auch hier Fälle vor, aus denen hervorgeht, daß dem Gesetz nur infolge strikter Durchführung und nicht infolge freiwilliger Befolgung Genüge geschehen wird. Unglücklicherweise ist es bisher nicht möglich gewesen, mehr als einen sehr geringen Bruchteil der importierten Artikel zu konspizieren, so daB es vorkommen kann, daB ein Artikel, welcher zu einer Zeit oder in einem Hafenort zugelassen worden ist, zu anderer Zeit oder an einem anderen Ort ausgeschlossen wird. Dies hat natürlich den Anschein einer Bevorzugung, während es in Wirklichkeit nur auf der Unmöglichkeit beruht, eine vollständige Kontrolle durchzuführen. I).

\section{Über die Stellung des Ventilators und iiber einige neuere Fortschritte beim Bleikammerprozeß.}

\section{Von E. Harminan und F. Benker. (Eingeg. d. 16./12. 1905.)}

Die jüngsten Veröffentlichungen in dieser Zeitschrift, námentlich von $L \ddot{u} \mathbf{t} y$, Dr. $N$ e u m a n n , Dr. $\mathrm{R}$ a a b e und Dr. S c h li e b s geben uns Veranlassung, zunächst nochmals unsere Ansicht bez. der Stellung des Ventilators beim Bleikammerprozeß zu präzisieren.

Wir sprachen uns früher bereits (diese Z. 16, $862-863,1903$ ) über diese Frage aus. Bei der lebhaften Polemik aber, die sich über diese Angelegenheit in der jüngsten Zeit eatwickelt hat, und bei den Schlußfolgerungen, die hieraus von verschiedenen Seiten gezogen worden sind, dürften unsere nachstehenden Mitteilungen über die Vorteile, die uns zur Stellung des Ventilators an das Ende des Systems und bei Vorhandensein von zwei $G$ a $y$ L u s s a c-Türmen zwischen diese oder bei nur einem G a y-Luss a c-Turm zwischen diesem und der letzten Bleikammer bestimmt haben, von allgemeincrem Interesse sein.

Bekanntlich plädiert die Mehrzahl der oben aufgeführten Autoren für die Aufstellung dieses Apparates v o r $\mathbf{n}$, und zwar entweder zwischen dem Kiesofen und dem Glover-Turm, oder zwischen diesem und der ersten Kammer des Bleikammersystems, oder endlich nach $L$ ü ty und nach $\mathbf{N}$ i ed e $\mathbf{n}$ ü h r zwischen zwei $G$ lover. 'Türmen, von welchen der eine den Namen ,Denitrifikator" erhalten hat.

Nach dem L ü t y schen Vortrage, der die letztere Stellung bespricht, muß der der Schwefelsäureindustrie fernstehende die Therzeugung gewinnen, daß durch diese Anordnung der BleikammerprozeB in ein vollkommen neues und bis dahin ungeahntes Stadium der Vollendung geleitet sei. Wir wollen dic Frage, ob dieses tatsächlich der Fall ist, heute nicht erörtern und lassen wir es auch dahin gestellt, ob die von $\mathrm{L} \ddot{\mathrm{u}} \mathrm{t} \mathrm{y}$ angegebenen Betriebszahlen dauernd und ohne Beeinträchtigung der Lebensdauer des Systems erreicht werden, da hierüber kaum maßgebende Resultate vorliegen können.

Dr. $R$ a a be weist hingegen in seiner beachtenswerten Abhandlung nach, daß für den Kammerbetrieb mittels Ventilator nicht die Erzeugung eines gewisson Uberdrucks oder Unterdrucks gegenüber der Atmosphäre maßgebend sei, sondern a 1 le in die erzielte Geschwindigkeit und Regelmäßigkeit, d. h. mit anderen Worten, die richtige Zusammensetzung der Gase, und ferner, da $\beta$ die Reaktion unter dem Uberdruck im wesentlichen dieselbe sei, als beim Unterdruck. Er folgert hieraus mit Recht, daß die Stellung des Ventilators in keiner Weise einen EinfluB auf den Verlauf der Reaktionen ausübe, daß sich die von $\mathrm{L}$ ï $\mathrm{t} y$ angeführten Zahlen aber leichter aus der Dimensionierung der Apparate ableiten lasse, sowie aus der Betriebsführung und Kühlung.

Wir unterlassen es, auf die Frage des Überdrucks in den Bleikammern hier nochmals näher einzugehen, da wir uns hierüber bereits in unserer früheren, oben zitierten Abhandlung eingehend geäußert haben, und da diese Frage ebenfalls in dem jüngsten Aufsatze des Herrn Dr. Ne u m a n n und in dem von Dr. $\mathbf{R}$ a a b e eingehend kritisiert wurde; nur möchten wir nicht verfehlen, darauf hinzuweisen, daß die in dem $\mathrm{N}$ e u m a n n schen Auf- 
satze zum Ausdruck gebrachten Befürchtungen bez. der $\mathrm{L}$ e be n s d a u e r eines derart mit Uberdruck arbeitenden Kammersystems sich bei einer uns bekannten, nach $\mathrm{N}$ i e d e $\mathrm{n} f$ ï $\mathrm{h} \mathrm{r}$ scher Methode arbeitenden Anlage als voll begründet erwiesen haben. Wie es sich mit etwaigen anderen, nach diesem System arbeitenden Anlagen verhält, entzieht sich unserem Urteil.

Die Stellung, welche wir dem Kammerventilator geben, ist, wie bereits oben erwähnt, nach wie vor $h$ in te $n$ am Ende des Systems und zwischen den beiden G a y-L u s s a c-Türmen, resp. bei nur einem Turm mit der oben angegebenen Variante. Die Gründe, welche uns zur Wahl dieses Platzes bestimmten, wurden bereits in der mehrfach von uns zitierten Abhandlung eingehend besprochen, und haben sich diese in der Zwischenzeit in zahlreichen weiteren Anlagen als durchaus gerechtfertigt erwiesen.

Wir gehen sogar einen Schritt weiter, und sind wir der Uberzeugung, daß die Aufstellung des Ventilators vorn und vor dem G 1 o v e $\mathrm{r}$ - Turm nutzlos, unlogisch und in manchen Fällen sogar gefährlich ist. Anders verhält es sich bei mechanischen Röstanlagen, bei welchen man erst - bedingt durch die große Anzahl derartiger Öfen, welche auf ein und dasselbe System arbeiten - durch Einführung des Ventilators direkt hinter demselben die sehr günstigen Resultate erzielt hat, die jetzt namentlich mit den $H$ ex resh off-Öfen vorliegen; aber auch in diesem Falle sehen wir hinten und am Ende des Systems einen zweiten Ventilator vor, wie das übrigens von Falding auch geschieht.

Tatsächlich ist bei einem rationell konstruierten und mit Malétra- oder Stückkiesöfen arbeitenden System der Auftrieb der heißen, von den Ôfen kommenden Gase so groß, daß derselbe in der letzten Kammer einen leichten Uberdruck erzeugt, der durch den Widerstand hervorgerufen wird, welcher durch die Gay-Luss a c-Türme gegeben ist. Um diesen zu überwinden, muB der vor dem G lov e r - Turm aufgestellte Ventilator einen beträchtlichen Druck in den Kammern ausüben, und zwar zum großen Nachteil der Bleiwände der letzteren: wir sind der Ansicht - und die Erfahrung hat dies in der oben erwähnten mit starkem Úberdruck arbeitenden Anlage vollauf bestätigt - , daß bei den von $\mathrm{L} u ̈ \mathrm{t} y$ angegebenen Grenzen die Lebensdauer eines derartigen Apparates auf die Hälfte zurückgedrängt wird.

Hierbei ist ferner zu beachten, daß sich bei dieser Anordnung auch der EinfluB von Wind und Wetter unfehlbar in unangenehmster Weise bemerkbar macht; jeder Schwefelsäurefabrikant weiß, in wie weit die atmosphärischen Einflüsse die Zugverhältnisse des Schornsteins beeinflussen, und ferner, wie dadurch die rationelle Leitung eines Kammersystems erschwert wird.

Des Ferneren weisen wir darauf hin, da $\beta$ durch die Aufstellung des Ventilators vorn bei ungenügenden, und für diese Anordnung nicht ganz besonders konstruierten Staubkammern dem G lo v e r Turme große Quantitäten von Flugasche zugeführt werden, ja daß diese sogar bis in die Kammern gelangen. Es sind, um diesen Utbelständen vorzubeugen, bekanntlich verschiedene Vorschläge gemacht, und verweisen wir bez. dieses Punktes auf den A. P. O'Bri e n schen Staubabscheider und auf die Anordnung besonderer sogenannter Cyklone, welch letztere auf einem ähnlichen Prinzipe beruhen, und die zwischen den Ofen und dem Glove r Turn eingeschaltet werden. Da diese Konstruktionen fast durchweg aus Amerika stammen, in welchem Lande bekanntlich die Anordnung des vorderen Ventilators in Gebrauch ist, so dürfte dieses ein Beweis dafür sein, daß sich der beregte Ubelstand auch dort in hervorragendem Maße gezeigt hat, wie das nicht anders möglich ist.

Durch diese großen Mengen von Flugasche sättigt sich aber die Berieselungssäure des $\mathrm{G} 1$ o v e $r$. Turms sehr schnell mit ersteren, wodurch eine ungenügende Wiedergewinnung der Nitroseverbindungen in den $G$ a y - L u s s a c - Türmen, damit ein vermehrter Verbrauch an Salpeter, und ferner ein schnellerer Verschleiß des G lover-Turms bedingt ist. Dieser Staub wird, wie gesagt, unter Umständen selbst bis in die Kammern befördert, wo er die erzeugte Kammersäure mit Eisen-, und eventl. mit Arsen- und Selenverbindungen verunreinigt und dieselbe dadurch für viele Zwecke unverwendbar macht. $\mathrm{Ob}$ die berührten Anordnungen zur Beseitigung dieses Ubelstandes v o l l e $n$ Erfolg verbürgen, entzieht sich unserem Urteil; das mit uns liierte Bureau des Herrn Peter S. Gilchrist Charlotte N. C., U.S. A., versichert uns dieses. Auf jeden Fall verteuern diese Einrichtungen aber den Bau einer Schwefelsäurefabrik, und sie lassen sich vermeiden, da man denselben Zweck durch die von uns gewählte Anordnung des Ventilators erreicht. Durch letztere sichert man sich aber noch verschiedene Vorteile, die wir nachstehend in Kïrze berühren.

1. haben wir es bei der Stellung des Apparates zwischen den beiden $\mathrm{G}$ a $y$ - $L$ u s s a c - Türmen, und nuchdem die Gase den ersten dieser Türme passiert haben, der ihnen die Nitroseverbindungen entzogen hat, nur mit einigen Prozenten an Sauerstoff, mit größeren Quantitaten an Stickstoff und mit geringen Spuren von Säuredämpfen zu tun, und ferner sind diese Gase kalt und trocken, wodurch eine größere Haltbarkeit des Ventilators bedingt ist.

2. läßt sich der Apparat an dieser Stelle ohne Sohwierigkeit auf den Boden unter den Kammern aufstellen, da man bei den Gay-LussacTürmen wohl immer genügend Platz hierfür hat. Es läßt sich also die Einschaltung des Ventilators auch bei vorhandenen Systemen stets ausführen, während dieses bei einer Einschaltung vorn und bei bestehenden Anlagen durch die Nähe der Kiesöfen, der Reservoire, der Hebevorrichtung für die Berieselungssäuren und durch die sonstigen Betriebsmanipulationen, die sich hier abspielen, häufig seine Schwierigkeiten hat, ja in manchen Fällen unmöglich ist.

3. kann man den Ventilator samt den erforderlichen Verbindungsrohren während des Betriebes montieren, da die erforderlichen Lötungen an $k$ a $l$ t e $n$ und dazu an sauren Gasen armen Stellen vorzunehmen sind, was beim G l o v e r - Turm bekanntlich nicht der Fall ist.

4. bietet die Aufstellung des Ventilators hinten bei späteren Reparaturen und bei undichten Stellen in den Kammerwänden und in den Decken den Vorteil, daß die erforderliche Auswechslung schnell 
und leicht auszuführen ist, während dieses bei einem mit Uberdruck arbeitenden System seine großen Schwierigkeiten haben dürfte.

5. bewerkstelligt sich die eventl. Ausschaltung des Ventilators an dieser Stelle in leichtester und bei der von uns gewählten Konstruktion in einfachster Weise, wie aus nachstehender Skizze ersichtlich ist; auch hier sprechen die kalten und an Säuredämpfen armen Gase erleichternd mit.

Stellung der Leitumgen beim Betrieb mit Ventilator.
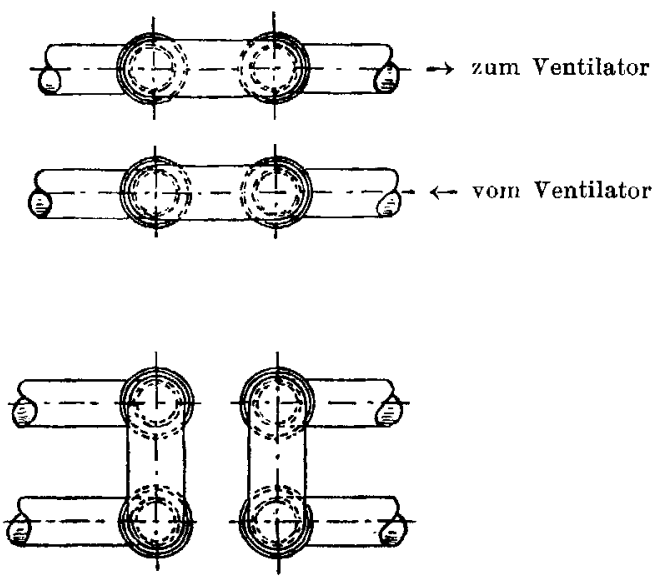

Stellung der Leitungen bei ausgeschaltetem Ventilator.

6. ist der Ventilator hinten ein idealer Regulierapparat, der die durch die Gay-Lussac Türme erzeugten Widerstände, und ferner auch jeglichen EinfluB, der durch Wind und Wetter hervorgerufen ist, vollkommen und in denkbar einfachster Weise aufhebt. Dabei ist der von uns gewählte Apparat in seiner Anschaffung billig und von langer Lebensdauer.

Wir haben nach dieser Anordnung, also mit der Einschaltung des Ventilators hinten, jetzt ca. 150 Kammersysteme in allen Größen und in allen Industrieländern und häufig in den kompliziertesten Ausführungen, soweit es sich um alte von uns rekonstruierte Kammersysteme handelt, in Betrieb, mit denen wir bei sachgemäßer Leitung des Betriebes seit Jahren die ausgezeichnetsten Resultate erzielt haben. Es liegt also für uns kein Grund vor, diesen Standpunkt $\mathrm{zu}$ verlassen und unseren Klienten andere Anordnungen zu empfehlen, von denen wir überzeugt sind, daß sie bei späterem Betriebe nicht so funktionieren, wie es unsere Einrichtung und unsere Anordnung tut.

Wir unterlassen es, auf die $\mathrm{N}$ i ed e $\mathbf{n} f$ ï $\mathrm{h} \mathbf{r}$ sche Aufstellung des Ventilators zwischen dem G lover-Turm und dem Denitrifikator heute näher einzugehen, da dies ja, wie bereits mehrfach von uns erwähnt, durch Dr. N e $\mathbf{u} \mathbf{m}$ a $\mathrm{n} n$ und Dr. $R$ a $a b$ e und neuerdings durch Dr. S o h liebs geschehen ist. Die Einwände des letzteren $g$ e g e $n$ unsere Aufstellung möchten wir jedoch mit einigen Worten berühren, soweit dieses ohne nähere Kenntnis der von ihm angeführten Kammersysteme möglich ist.

Wenn das von Dr. Sch liebs aufgeführte System A(diese Z. 18, Heft48 [1905] bezüglich der Ófen und Kammern an der Grenze seiner Leistungsfähigkeit angelangt war, so kann selbstverständlich auch der Ventilator keinen durchschlagenden Erfolg und keine Erhöhung der Produktion, wozu in erster Linie eine erhöhte Zufuhr von schwefliger Säure erforderlich ist, ergeben. Es ist uns deshalb unverständlich, wie Dr. S c h li e b s gerade d i e s e Anlage gegen die Anordnung eines Ventilators hinten aufführt. - Wir vermuten übrigens, da.B sich die dieses System betreffenden Mitteilungen auf eine niederrheinische Schwefelsäurefabrik beziehen, welche wir selbst vor einigen Jahren besichtigt haben, und sollten unsere Vermutungen richtig sein, so können wir nur konstatieren, daß3 zurzeit unseres damaligen Besuches der Ventilator vollkommon verkehrt aufgestellt war.

Die Verhältnisse des von Dr. S chl i e bs auf der gleichen Seite aufgeführten Systemes B entziehen sich nach den dürftigen Angaben darüber jeglichen Urteils; wenn aber tatsächlich die Produktion resp. Kiescharge nach Einschaltung des Hart. bleiventilators $b$ vor dem G a y-Lussac Turm um 20-25\% sank und der Salpeterverbrauch um $30-40 \%$ stieg, so behaupten wir - und wir zweifeln nicht daran, daß uns jeder Schwefelsäure. techniker, welcher mit unseren diesbezüglichen Einrichtungen gearbeitet hat, darin zustimmen wird -, daß sonstige schwerwiegende Fehler in der Anlage oder in der Betriebsleitung obwalteten, die diese Resultate bedingt haben.

Wir plazieren den Ventilator a u s s o h li e B. li $\mathrm{ch}$, wie bereits mehrfach betont, an die ron Dr. Schliebs aus ,theoretischen und praktischen Erfahrungen für die am wenigsten günstig" bezeichnete Stelle; leider werden diese theoretischen Gründe nicht näher motiviert, und können wir Herrn Dr. S c h li e bs daher nur erwidern, daB wir in allen Anlagen, die wir ausführten, bei sach. gemäßer Leitung mindestens $20-30 \%$ Mehrleistung. häufig sogar eine solche von $80-100 \%$ erreicht haben.

Wenn Dr. S ch li e b s eine rheinische Fabrik mit Ventilator vor dem G lover-Turm und in Verbindung mit Herresh off-Ofen sehr gut arbeiten sah, so dürften wir wohl nicht fehlgehen, wenn wir vermuten, daß es sich um ein sehr großes Werk in unserer allernächsten Nachbarschaft handelt. Ist diese Voraussetzung richtig, und hätte Herr Dr. S c h li e bs sich dann näher erkundigt, so dürfte er bemerkt haben, daß auch dieses System mit einem Ventilator hinten zwischen den $\mathbf{G}$ a $\mathbf{y}$ L u s s a c-Türmen arbeitet. Dieser Ventilator wurde vor der Aufstellung der $\mathrm{Her}$ e s h of $\mathbf{f}$ Ofen bei dem betreffenden System eingeschaltet, und stieg hierdurch und in Verbindung mit unserer Wasserzerstäubung die Leistung desselben um ca. $85 \%$ bei gleichzeitiger, nicht unwesentlicher Reduktion des Salpeterverbrauchs.

Wir möchten dann noch in nachstehendem auf einige $\mathrm{N}$ e u e $\mathbf{r}$ u $\mathrm{n}$ g e $\mathrm{n}$ hinweisen, die wir beim Bleikammorprozesse eingeführt haben, und die allgemeineres Interesse finden dürften, zumal die Schwefelsäurefabrikation bei den teilweise sehr gedrückten Preisen der Säure und bei der enormen Zunahme des Bedarfs an Schwefelsäure von großer Wichtigkeit geworden ist. 
Nur diejenige Fabrik kann unseres Erachtens unter den heutigen Bedingungen und Verhältnissen konkurrenzfähig bleiben, welehe durch ihre Einrichtungen in der Lage ist, ihre Generalunkosten und die Verzinsungs- und Amortisationsquoten, auf die erzeugte Säure verrechnet, auf ein Minimum zu beschränken: dieses ist aber nur durch den Intensivbetrieb möglich.

Die wesentlichsten Punkte, auf denen dieser beruht, und durch deren Befolgung wir in unseren Einrichtungen nachweislich Leistungen von $8-10 \mathrm{~kg}$ Kammersäure von $53^{\circ}$ Bé. per cbm und innerhalb 24 Stunden erzielen, lassen sich wie folgt zusammenfassen:

1. Rationellste Konstruktion der Kammern, der Türme und ihrer Verbindungen untereinander.

2. Wasserzerstäubung in den Kammern und vollkommenster Ersatz des bisher angewendeten Wasserdampfes durch kaltes zerstäubtes Wasser.

3. Aufstellung des Ventilators, um die Geschwindigkeit der Gase und damit deren Zusammensetzung zu regeln.

4. Exakteste, genau dem jeweiligen Bedarf angepaßte Zuführung von Salpetersäure resp. von Salpeter in Lösung durch Zerstäubung.

5. Künstliche Kühlung der Endgase der letzten Kammer vor ihrem Eintritt in den Gay - L u s sac Turm, unter gleichzeitiger Kondensation der in diesen Endgasen enthaltenen Wasserdämpfe.

6. Intensive und natürliche Kühlung der Kammerwände.

7. Einrichtung von zwei e r s te n Kammern anstatt der bisher üblichen einen.

Uber die Punkte 1-3 dürften sich weitere Mitteilungen erübrigen, da die Vorteile, welche in den Konstruktionen der einzelnen Teile eines Kammersystems, und in den beiden Punkten 2 und 3 begründet sind, jetzt wohl von allen Schwefelsäuretechnikern anerkannt werden. Im übrigen haben wir uns über die hierin liegenden Vorteile auch schon in dieser $Z$. 16, 1903 eingehend geäußert. Wir haben diese Mitteilungen und speziell diejenigen iiber die Wasserzerstäubung ferner durch eine kleine Broschüre im September v. Js. ergänzt, in der wir die Fortschritte besprachen, die wir seit unseren früheren Veröffentlichungen in der Konstruktion der Wasserzerstäuber gemacht, und die uns in die Lage gebracht haben, den bisherigen Wasserdampf in a lle $\mathbf{n}$ Kammern eines Systems durch kaltes Wasser zu ersetzen, und damit bei Neuanlagen den Dampfkessel gänzlich zu beseitigen, soweit es sich um den eigentlichen Schwefelsäurebildungsprozeß handelt. Wir wiesen in dieser Broschüre, welche Interessenten auf Wunsch gern zur Verfügung steht, an einem Systeme von einer täglichen Leistung von 20 t Kammersäure von $53^{\circ}$ Bé. rechnerisch nach, daß sich durch diese Verbesserungen für ein derartiges System neben einer Mehrproduktion von 5 t Kammersäure täglich, eine Ersparnis von M 9-10 000 per Jahr ergibt. Wir sind in der Lage, diese Resultate bei vielen Anlagen nachzuweisen, sowie unsere Mitteilungen durch Zeugnisse erster Firmen zu erhärten, in denen durchweg diese Vorziige anerkannt und die vollste Zufriedenheit über dic erzielten Resultate zum Ausdruck gebracht werden. Auch in den Vereinigten Staaten, in denen die Wasserzerstäubung noch neu war, und in der Zwischenzeit durch uns und in Verbindung mit dem Bureau des Herrn Peter S. Gilohrist eingeführt wurde, haben unsere Einrichtungen ausgedehntesten Eingang gefunden, und selbst das konservative Großbritannien verschließt sich nicht mehr gegenüber den Vorteilen, welche in unserer Wasserzerstäubung und in dem Ventilatorenbetrieb begründet sind.

Wir gehen dann zu Punkt 4, die $\mathrm{E}$ i n $\mathrm{f} \ddot{\mathrm{u}} \mathrm{h}$ rung der Salpetersäure, resp. der Sal peter lös ung, über.

Der gute Gang des Kammersystems wird bekanntlich sehr von der gleichmäßigen Zuführung der Salpetersäure in den als Denitrifikator dienenden G 1 o v e r - Turm beeinflußt. Nun wandte man früher, und wendet man auch jetzt noch in der Mehrzahl der Schwefelsäurefabriken für diesen Zweck eine Mariotté sche Flasche oder ein anders gestaltetes Gefäß mit Hahn an, aus welchem die Salpetersäure dem G l o v e r - Turm durch ein durch die Decke reichendes Trichterrohr zugeführt wird. Diese Art der Einführung hat den Nachteil, daß die Menge der durch den Hahn ablaufenden Säure durch den jeweiligen Säurestand in dem Gefäß beeinflußt wird, was bei der verhältnismäßig geringen Menge, um die es sich handelt, nicht ohne Bedeutung ist, und ferner versetzt sich der Ablaufhahn mit etwaigen, in der Salpetersäure enthaltenen Verunreinigungen sehr leicht, wodurch die $\mathrm{Zu}$ führung der Salpetersäure zeitweise ganz unterbrochen werden kann, wie das jedem Schwefelsäuretechniker bekannt ist.

Wir vermeiden diese unliebsamen Störungen dadurch, da $\mathrm{B}$ wir 3 durch Glasheber miteinander verbundene Gefäße neben der Glover.Turmdecke aufstellen, von denen das erste zur Ein- und Nachfüllung der Säure und das zweite als Filter dient; die filtrierte Säure gelangt dann durch einen dritten Heber in das letzte Gefäß, von welchem sie durch ein besonders gebogenes Glasrohr, zum Trichterrohr in der G 1 o v e r - Turmdecke abläuft. Dieses Glasrohr ist dadurch graduiert, daß sein Ablaufschenkel an seinem Ende zusammengezogen und auf eine geringere Offnung reduziert worden ist. Wir sehen von diesen graduierten Schlußhebern mehrere, vorher genau auf die per Zeiteinheit ablaufende Säuremenge geeichte vor, und sind wir durch Wechseln dieser Heber in der Lage, die jeweilig für den Gang des Prozesses erforderliche Menge per Zeiteinheit mit mathematischer Genauigkeit einzustellen, und dadurch die Zuführung auch tatsächlich in präzisester Weise zu erreichen.

Uberall, wo wir dieses System der Zuführung der Salpetersäure einführten, haben wir nicht nur eine vollkommene Regelmäßigkeit in den Reaktionen, sondern auch eine wesentliche Verminderung des Bedarfș an dieser Säure konstatieren können; in einzelnen Fabriken betrug diese 0,4\% vom ersten Tage ab, und zwar bei Systemen, welche auch vorher nicht allzu ungünstig arbeiteten.

In denjenigen Fabriken, in denen die Frage der Reinheit der erzeugten Schwefelsäure weniger von Bedeutung ist, und in welchen dieselbe, unbeschadet ihrer späteren Verwendung, einige Spuren 
von Bisulfat enthalten darf, empfehlen wir die Zerstäubung einer Salpeterlösung von ca. 5-8 $8^{\circ}$ Bé.

Wir benutzten hierfür früher den sogenannten P otut-Apparat, sind hiervon jedoch seit ca. 2 Jahren abgekommen, weil dieser Apparat den Nachteil hat, daß zur Zerstäubung Dampf in Frage kommt, der dazu noch an einer Stelle eingeführt wird, an der ohnehin schon genügend Dampf vorhanden ist, und an welcher man dem BleikammerprozeB Wärmeeinheiten entziehen sollte, anstatt ihm solche zuzuführen.

Diese Ubelstände haben wir dadurch beseitigt, daB wir die Zerstäubung der filtrierten Salpeterlösung nach dem gleichen Prinzipe und durch komprimierte Luft ausführen, nach welchem wir bei unserer Wasserzerstäubung für den Bleikanmerprozeß arbeiten. Auch die bei ersterer in Frage kommende Apparatur ist, abgesehen von einigen Modifikationen, die gleiche; die Zerstäubung selbst erfolgt in der ersten Kammer.

In verschiedenen nach dieser Methode arbeitenden Fabriken von einer Leistung von $8 \mathrm{~kg} 53 \mathrm{er}$ Säure beträgt der nittlere Salpeterverbrauch nicht mehr als $0,45 \rightarrow 0,50 \%$, wodurch die Unkosten für den letzteren auf M 0,10 pro $\% \mathrm{~kg}$ erzeugte Säure herabgedrückt worden sind.

Punkt 5. Künstliche Kühlungder Endgase der letzten Kammer vor ihrem Eintritt inden Gay-LussacTurm unter gleichzeitiger Kondensation der in diesen Endgasen enthalten en. Wasserdämpfe.

$\mathbf{L}$ ü $\mathrm{t}$ y hat sich in seinem Vortrage in Bremen (d. Z. 18, 1256 [1905]) veranlaßt gesehen, diese von uns eingeführte Kühlung der Endgase einer wenig wohlwollenden Kritik zu unterziehen. Befremdend ist es dieser Kritik gegenüber, daß $\mathrm{N}$ i e d e $\mathrm{n} \mathbf{f} \ddot{\mathrm{u}} \mathbf{h}$ dann diese Gaskühlung in dem von ihm vorgeschlagenen Kammersystem (18, Fig. 4, 1262), in welchem das enorme Quantum von 80 tons Kammersäure von $50^{\circ}$ Bé. per Tag dargestellt werden soll, doch vorsieht, ja unseren Apparat anscheinend genau kopiert - Wir dürften danach in der Annahme wohl nicht fehlgehen, daß Genanntem nachträglich der Gedanke gekommen sein dürfte, daß doch wohl etwas Gutes an dieser Kühlung sein könne, daß es aber, um dem eigenen Widerspruche zu begegnen, angezeigt sei, unserem Apparat einen Plattenturm hinzuzufügen. Wir verkennen die vorzüglichen Eigenschaften dieses letzteren Apparates nicht, ob der Effekt durch diesen in gleich einfacher und vollkommener Weise erreicht wird, wie durch unseren, in seiner Anschaffung sehr billigen Apparat, möchten wir aber bezweifeln.

Es ist eine bekannte Tatsache, daß die von den Endreaktionen in der letzten Kammer abgeleiteten Gase namentlich während der heißen Sommermonate eine ziemlich hohe 'Temperatur zoigen, und daß dieselben überdies eine mehr oder minder große Menge von Wasserdampf aufweisen, die in den G a y - L u s s a c - Turm geleitet, die $60^{\circ}$ Bé. Berieselungasäure desselben unfehlbar verdünnen, und dadurch eine schlechte Wiedergewinnung der Nitroseverbindungen herbeiführen. Es müssen daher diese beiden Ubelstände unbedingt beseitigt werden, und war dieses die Erwägung, welche uns zur Konstruktion des in Frage stehenden Rippen- rohrkühlers veranlaßte, den wir trotz $\mathbf{L}$ ü $\mathbf{y}$. $\mathrm{N}$ i ed e $\mathrm{f}$ ü $\mathrm{h} \mathbf{r}$ seit einer Reihe von Jahren mit bestem Erfolge anwenden. Wir können Genannten daher diese Anordnung nur auf das wärmste empfehlen, und geben wir ihnen im voraus die Versicherung, daß sie ihre bisherige Ansicht sicherlich ändern werden, vorausgesetzt, daß die Anwendung des Apparates in richtiger und sachgemäßer Weise erfolgt.

Wir schalten den Apparat zwischen dor letzten Kammer und dem ersten $\mathrm{G}$ a $\mathrm{y}-\mathrm{L}$ u s s a c - Turm ein. Die Konstruktion desselben ist schon durch den Namen angedeutet, den wir dem Apparat gegeben haben. Eine größere Anzahl von Bleirohren bestimmten Querschnitts, die zur Erhöhung ihrer Kühlfläche nach außen hin mit Rippen versehen sind, und deren Gesamtquerschnitte in einem bestimmton Verhältnis zu dem jeweilig für das be. treffende System in Frage kommenden Gasquantum stehen, sind dadurch zu einem an sich geschlossenen Apparat vereinigt, da $\beta$ an beiden Enden der gesamten Rippenrohre Bleikästen von bestimmten Querschnitts- und Höhenverhältnissen angeordnct sind, und zwar derart, daß die Rippenrohre mit einer gewissen Neigung in die Stirnwand des einen Kastens einmünden: durch diese Neigung der Rohre ist das in denselben und aus den Endgasen kondensierte Wasser gezwungen, nach dem betreffenden Kasten abzulaufen, aus welchem es dann selbsttätig entfernt wird. Das gesamte vorstehend skizzierte Kühlsystem ist in einen großen mit Wasserkühlung ausgerüsteten Bleikasten eingebaut. Die aus der letzten Kammer kommenden Endgase treten in den einen der Röhrenkästen ein, dureh. streichen die Rippenrohre und treten dann aus dem anderen Endkasten, von ihrer Fcuchtigkeit befreit und in abgekühltem Zustande, zum ersten $\mathbf{G}$ a $\mathbf{y}$. L us s a c-Turm über, der mit den nach vorstehender Weise vorbereiteten Gasen seine Funktionen in idealer Weise erfüllt.

Punkt 6. Intensive und nat ür. liche Kühlung der Kammerwände.

Um die Kondensation der Säure im Verhältnis zu ihrer Bildung in den Bleikammern zu befördern. und um einen Teil der Wärmeeinheiten, welche sich infolge der Reaktionen der Kammergase untereinander entwickeln, abzuleiten, konstruieren wir die Kammern derart, daB etwaige Hindernisse in der Berührung der äußeren abkühlend wirkenden Luft mit den Bleiwänden nach Möglichkeit vermieden werden. Wir schließen zur Erreichung dieses Zieles sämtliche Querriegel aus und ordnen die mit einer Ecke rechtwinklig zum Kammerblei aufgestellten vertikalen Kammerpfosten in Abständen von 1,50-1,70 $\mathrm{m}$ zueinander an. Die Wände selbst und auch die Decken hängen wir durohweg an Rundeisen auf, über welche sich beiderseitig mit dem Kanmerblei verlötete Bleilappen legen, so daß die äußere Luft auch hier überall und ungehindert leicht cinwirken kann.

Wir untergtützen endlich die Abkühlung der Wünde in bekannter Weise dadurch, daß wir die Fußböden der Kammergänge aus Latten herstellen, durch deren $Z$ wischenfugen die Luft frei hindurch treten kann. Zur Beförderung dieses an den Bleiwänden ständig emporstreichenden Iuftstromes versehen wir dann, in ebenfalls bekannter Art, das 
Kammergebäude mit einem auf seine ganze Länge verlaufenden Dachreiter.

Punkt 7. Einriohtungvon 2 ersten Kammern anstatt der bisher üb lichen einen.

In einem aus 3 Kammern bestehenden System haben wir bei normalem Gange desselben ca $75 \%$ der Gesamtleistung in der ersten Kammer, ca. 20\% in der zweiten und ca. $5 \%$ in der letzten Kammer; die Leistung per cbm ist danach und naturgemäß in der ersten Kammer eine ganz wesentlich größere als in den beiden anderen. Hierdurch erwächst aber bei großen Systemen mit hoher Produktion der Nachteil, daß die Temperaturen in der ersten Kammer trotz Wasserzerstäubung und trotz natürlicher Kühlung der Kammerwände und Decken unvorteilhaft werden können, namentlich ist dieses in den heißen Sommermonaten oder in Ländern mit heißem Klima der Fall.

Dieses hat uns bewogen; anstatt der bisherigen einen ersten Kammer, deren zwei erste anzuwenden, auf welche wir dann die vom G lover-Turm kommenden Gase gleichmäßig verteilen. Auf diese Weise erzielen wir eine wesentlich intensivere Produktion, reduzieren den Salpeterverbrauch und haben den weiteren Vorteil der bedeutend reduzierten Temperatur.

Die u. a. in Nantes erreichten Leistungen bis zu $10 \mathrm{~kg} \mathrm{Kammersäure} \mathrm{von} 53^{\circ}$ Bé. und darüber per cbm, und zwar während der Sommermonate, gaben uns den Beweis, da $B$ unsere Erwägungen durchaus richtige waren, und daß man auf diese Weise ohne Erhöhung des Anlagekapitals und auf leichteste Art zu bis dahin unbekannten Resultaten kommt, und zwar, was wir besonders betonen möchten, davernd, und ohne eine frähzeitige Beschädigung der Bleiwände befürchten zu müssen.

Schließlich möchten wir noch auf die überaus günstigen Resultate hinweisen, welche mit den mechanischen Röstöfen erzielt worden sind, Resultate, welche diesen letzteren immer mehr den Vorrang vor den Handöfen sichern. Um so befremdlicher ist es, daB $\mathrm{L} \ddot{\mathrm{u}} \mathrm{t} \mathrm{y}$ auch hier wieder zu anderen Resultaten kommt. Seine diesbezüglichen Ausführungen wurden in der $Z$ wischenzeit von $E$. W. $\mathrm{K}$ a uffmann (vgl. diese Z. 18, 1628 [1905]) widerlegt. Jeder Schwefelsäuretechniker, welcher mit gut konstruierten und namentlich auch richtig aufgestellten mechanischen Ofen arbeitet, wird $\mathrm{K}$ a $\mathbf{u f f m} \mathrm{m} n$ aus vollster Überzeugung zustimmen, daß die von ihm hervorgehobenen Vorteile dieser Ofen eher zu niedrig, als zu hoch bewertet sind: um so erstaunlicher ist daher obiges Uirteil.

Wir hoffen, daß wir mit unseren vorstehenden Ausführungen neue Anregung gegeben haben, um auf dem betretenen Wege der Vervollkommnung des Bleikammerprozesses weitere Schritte vorwärts zu machen, und denselben dadurch seiner höchsten Vollendung zuzuführen. Wir fassen dieses zu erstrebende Ziel dahin zusammen, die Einrichtung eines Bleikammersystems so zu gestalten, daß die Herstellungskosten und dic Generalunkosten, die Verzinsungs- und Amortisationsquoten auf die er- zeugte Säure berechnet, auf ein Minimum beschränkt bleiben, ohne daß der Apparat einem frübzeitigeren Ruin ausgesetzt ist, als es bei den gewöhnlichen alten Systemen der Fall war.

\section{Von welchen Gesichtspunkten sind geröstete Blenden hinsichtlich ihres Entschweflungsgrades zu beurteilen?}

\author{
Von V. Hassreidter-Trooz. \\ (Eingeg. d, 30,112, 1905.)
}

Bekanntlich gibt der Totalschwefelgehalt einer gerösteten Blende kein Kriterium ab, ob die Röstung gut oder schlecht ausgeführt wurde, und es kann beispielsweise der Fall eintreten, daß eine Blendesorte mit 3\% Totalschwefel im Röstgut als zufriedenstellend, eine solche mit $2 \%$ aber als ungenügend geröstet $\mathrm{zu}$ betrachten ist. Dies hat, wie weiter bekannt ist, darin seinen Grund, daß gewisse, fast nie fehlende Begleiter der Blenden, wie Blei (als Bleiglanz), Kalk, Magnesium und Baryum (als Carbonate oder als Sulfate), während der Röstung ganz oder teilweise in Sulfate verwandelt werden bzw. als solche im Röstgut verbleiben.

Der in Form von solchen Sulfaten zurückbleibende Schwefel kann einerseits für die Schwefelsäureausbeute nicht mehr in Betracht kommen, kann aber auch andererseits vom Konsumenten der gerösteten Blende nicht Grund einer berechtigten Reklamation sein. Von diesem Gesichtspunkte ausgehend, herrscht in manchen industriellen Bezirken die den Produzenten sowohl als auch den Konsumenten bindende Utbereinkunft, daß als ,,schädlicher" bzw. noch, ,austreibbarer" Schwefel die Differenz zwischen Totalschwefelgehalt (a) und der Summe des an die oben aufgeführten Elemente (als Sulfate gedacht) gebundenen Schwefels (b) zu verstehen ist. Dabei geht man stillschweigend von der übrigens noch nicht bewiesenen Annahme aus, daß die oben aufgeführten Elemente bei der Röstung vollständig in Sulfate übergeführt werden und auch nicht mehr zu entschwefeln sind.

Diese Ansicht finden wir z. B, auch in L u $\mathrm{n} g$ e s "Taschenbuch" vertreten, doch soll hier umsoweniger ein Vorwurf gegen dieselbe erhoben werden, als der Verfasser dieser Zeilen selbst einen bescheidenen Teil der Verantwortung dieser Angaben seinerzeit übernommen hat.

Halten wir an der oben gegebenen Definition des ,schädlichen" Schwefels vorläufig fest, so ergibt sich, daß die Differenz $a-b=c$ demjenigen Schwefel entspricht, der als Zinksulfatschwefel (d) und als Sulfidschwefel (e) noch im Röstgut verblieben ist. Es erhellt darans, daB $\mathrm{a}-\mathrm{b}=\mathrm{c}=$ $\mathrm{d}+\mathrm{e}$ ist.

Nebenbei bemerkt, sei hier von dem in gerösteten Blenden problematischen Vorhandensein von basischem Zinksulfat abgesehen, doch wollte ich auch diesen Punkt berührt haben.

Es fragt sich nun, ob es vorteilhafter ist, die Größe $c$ aus der Differenz von $a-b$ oder aus der Summe von $d+e$ zu bestimmen. 